\title{
The Process of Interpreting Images obtained by Scanning Electron Microscopy.
}

\author{
Patrick Echlin
}

Cambridge Analytical Microscopy, Cambridge, CB4 1XA, United Kingdom.

This paper will consider the ritual that must occur after the final stages of examining the unique SE and BSE images that arise from the interaction of a high-energy beam of electrons with a specimen studied in the SEM. This final process may be difficult to achieve, accurately, because mental processes in our brain carry it out and our interpretations will, consciously or unconsciously, be subjected to various biases. It is suggested that this interpretative process is quite different from the way we have learnt to view the natural environment we live in, and the way we decode pictures and drawings to translate any narrative they may contain. In addition, the images we obtain by the SEM are always a 2-D counterpart that complements the 3-D objects. These difficulties do not occur with numerical data that is always in the form of factual information presented in some arithmetical form.

Before we attempt to consider interpreting the SEM images, we first need to confirm the image acuity. This is the prime responsibility of the observer who views and/or records the image. We first need answers to the following questions. Is the image, which may well be unique, representative of the object from which it is derived? Is the transformation from the object to the image of sufficiently high fidelity? Are we aware of all the perturbation which may have been introduced by faulty preparation, instrumentation, examination, analysis and recording? Are we able to recognize, isolate and separate artefact from damage? Damage is defined as something that occurs as an unexpected event during preparation, examination and analysis. In contrast, artefacts arise as a consequence of deliberate attempts to alter the state of the object so it may be better imaged and analysed in the microscope. How much correlative microscopy has been carried out on the object? What additional non-optical information is available about the object?

Our first view of the image of an object is that displayed on the microscope TV screen. To avoid further beam damage, we usually quickly record this image in a digital form that can be easily transformed into a permanent photograph. It will be necessary to consider how much information is needed to interpret the image. Previous experience and context lessen the required pixel density. Unfamiliarity will require more pixels to provide more information. A photograph is accepted as a piece of ideological positive evidence and an objective representation of truth. Unlike our holiday snaps, scientific photographs should not be considered an emotional record of a cherished object that substantiates subjective feelings. Photographs are a very good way to record images. They are in strict geometric perspective and supply information within a conceptual framework. We consider the photograph to be irrefutable evidence. The photograph does not lie although the object may be false. Photographs preserve the look of things and although they may cut across a time line, they can be studied over time. Unfortunately, photographs have a number of failings. They are always of the past and may be ambiguous and non-representative if out of context. Electron beam images can only be expressed in amplitude contrast - we do not have green or red electrons. Transient effects of illumination may, unknowingly, limit the images we photograph and we must remember that they are always 2-D representations of 3-D objects. Although the photograph is a powerful way of recording images, we should, perhaps, not reject drawings entirely. They are a translation and expression of structural understanding that can be used in combination with photographs to 
condense and magnify information. We should consider comparing the unbiased objectivity of the photograph with the acuity, meaning, subjectivity and explanation of drawings.

One of the most attractive features of SEM images is that they appear to be in 3-D. They are immediately familiar to us because, like the natural world we live in, they generally appear illuminated from above. This feature, paradoxically, makes it either very easy, or very difficult to interpret and the viewer must constantly remember that the image is 2-D and there really is no "up" and "down" or "in" and "out". There are a number of techniques that can be used to further restore the information in the 2-D image, back towards the original 3-D of the object. Stereogrammetry is a simple matter of image processing and the use of stereo pairs enhances the apparent three dimensionality of the image Relief shadowing, computer graphics, serial sectioning and reconstruction, and the rigorous mathematical analysis by stereology, can all be used in this process.

Once we have cursorily examined the live image and, more thoroughly, examined the recorded image, how do we now proceed with the interpretation? The process of transferring the tangible visual image to the mind is complex and poorly understood. It will not be discussed further here. Our eyes look at parts of the image and then anticipate where next to look. The images are probably handled, interpreted, stored and recalled by non-verbal means, such as iconic thinking using a mental machine language. To illustrate this process, think of the mental image of "a warm summers day". In the process of interpretation, we look for the simplest and most economic patterns, we separate voids from solids and accept that things that are close together, probably belong together. Initially, we compare the unambiguous conscientious message of the new image with our pre-conceived view and see if the new image falls within the accepted consensual body of knowledge. For example, we interpret the new conscientious image as a mitochondrion because it fits, more or less, into the accepted canonical view of all mitochondria. The exciting point in microscopy comes when the conscientious and consensual messages do not quite agree. We need to be quite sure before we can say we are looking at something entirely new.

In spite of all the care we take looking at images, a number of examples of visual illusions will be shown to demonstrate how easy it is to arrive at a very confusing interpretation. The first and most familiar is the apparent 3-D appearance of the SEM image. Less familiar inconsistencies are mistaken hypothesis, depth ambiguity and reversal, imagined perspective and inferential illusions. Other illusions include figure ground reversals, imagined borders and the juxtaposition of lines. These illusions arise as a consequence of ignoring other information.

So in the end, how should we proceed to interpret an SEM image? There is, alas, no general easy answer because each of us has our own experience, criteria of excellence and anticipation of result. The following points may help in the process of interpretation. 1. We must make sure the image is devoid of any damage and that one is aware of any artefacts. 2. Previous experience and familiarity is always a good starting point. 3.We must be flexible in possibilities, explanations and projections. 4. We should learn to develop consistent hypotheses when ejecting miss-readings. 5. We must recognize that conflicts of evidence may either be a conflict of data or a paradox of perception. 6 . We must assemble as many different clues from seemingly disparate disciplines. 7 .We should not reject the aesthetic quality of the image. 